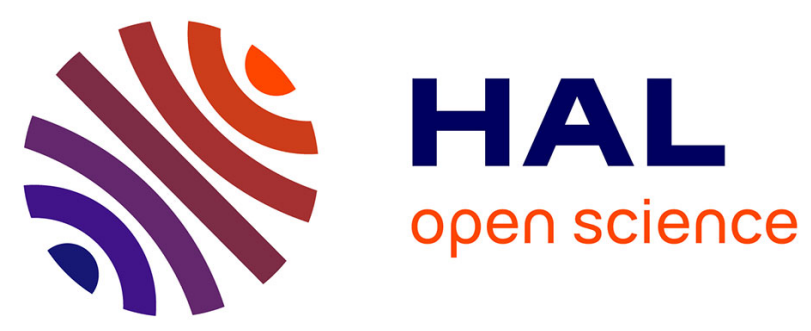

\title{
Ultrahigh-rectification near-field radiative thermal diode using infrared-transparent film backsided phase-transition metasurface
}

Yang Liu, Yanpei Tian, Fangqi Chen, Caratenuto Andrew, Liu Xiaojie, Mauro Antezza, Yi Zheng

\section{- To cite this version:}

Yang Liu, Yanpei Tian, Fangqi Chen, Caratenuto Andrew, Liu Xiaojie, et al.. Ultrahigh-rectification near-field radiative thermal diode using infrared-transparent film backsided phase-transition metasurface. Applied Physics Letters, 2021, 119 (12), pp.123101. 10.1063/5.0058779 . hal-03350625

\section{HAL Id: hal-03350625 https://hal.science/hal-03350625}

Submitted on 21 Sep 2021

HAL is a multi-disciplinary open access archive for the deposit and dissemination of scientific research documents, whether they are published or not. The documents may come from teaching and research institutions in France or abroad, or from public or private research centers.
L'archive ouverte pluridisciplinaire HAL, est destinée au dépôt et à la diffusion de documents scientifiques de niveau recherche, publiés ou non, émanant des établissements d'enseignement et de recherche français ou étrangers, des laboratoires publics ou privés. 


\title{
Ultrahigh-rectification near-field radiative thermal diode using infrared-transparent film backsided phase-transition metasurface
}

\author{
Yang Liu, ${ }^{\top}$ Yanpei Tian, ${ }^{\top}$ Fangqi Chen, ${ }^{\top}$ Andrew Caratenuto, ${ }^{\top}$ Xiaojie Liu, ${ }^{\top}$ Mauro Antezza, ${ }^{2,3}$ (D) and Yi Zheng ${ }^{1, a}$

\begin{abstract}
AFFILIATIONS
${ }^{7}$ Department of Mechanical and Industrial Engineering, Northeastern University, Boston, Massachusetts O2115, USA

${ }^{2}$ Laboratoire Charles Coulomb (L2C), UMR 5221 CNRS-Université de Montpellier, F-34095 Montpellier, France

${ }^{3}$ Institut Universitaire de France, 1 rue Descartes, F-75231 Paris Cedex 05, France
\end{abstract}

a) Author to whom correspondence should be addressed: $y . z h e n g @$ northeastern.edu

\begin{abstract}
We present a theoretical study of near-field radiative thermal rectification combining phase-transition and high-infrared-transmittance materials. The phase-transition material vanadium dioxide $\left(\mathrm{VO}_{2}\right)$, with a metal-insulator transition near $341 \mathrm{~K}$, is utilized under a reasonable temperature. Four types of high-infrared-transmittance materials, including potassium bromide, sodium chloride, polyethylene, and magnesium fluoride, are introduced as thin film substrates under a $\mathrm{VO}_{2}$ grating on one side of the near-field rectifier. We explore the effects of various high-infrared-transmittance thin-film substrates and relevant geometric parameters on the thermal rectification of the device. The results show that thermal rectification can be greatly enhanced by using a one-dimensional $\mathrm{VO}_{2}$ grating backed with a high-infraredtransmittance thin-film substrate. With the introduction of a high-infrared-transmittance substrate, the rectification ratio is dramatically boosted due to the enhancement of the substrate transmittance. This work predicts a remarkable rectification ratio as high as $161-$ greater than the recently reported peak values for comparable near-field radiative thermal rectification. The results outlined herein will shed light on the rapidly expanding fields of nanoscale thermal harvesting, conversion, and management.
\end{abstract}

The study of near-field radiative thermal rectification, which can preferentially facilitate radiative heat transfer in one direction through a micro/nanoscale gap, has recently garnered significant attention. ${ }^{.}$

Due to their contactless structures, radiative thermal rectification devices do not suffer the same limitations as conduction-based thermal rectifiers, which are restrained by both the speed of phonons and the presence of Kapitza resistances. ${ }^{8-14}$ Meanwhile, the near-field radiative heat transfer (NFRHT) between closely spaced objects can exceed the blackbody radiation limit by several orders of magnitude due to the tunneling of evanescent waves and coupling of surface phonons or plasmon polaritons, which provides near-field radiative thermal rectifiers with excellent thermal rectification performance. ${ }^{15-29}$ By taking advantage of the near-field radiative thermal rectification, devices like thermal diodes, ${ }^{4}$ transistors, ${ }^{9}$ switches, ${ }^{30}$ and thermal memories ${ }^{31}$ can be leveraged to attain highly efficient functionality in nanoscale heat control and thermal modulation.

To gauge the rectification efficiency of a thermal diode, we utilize the definition of the rectification ratio $R=\left(Q_{F}-Q_{R}\right) / Q_{R}$, where $Q_{F}$ and $Q_{R}$ refer to the forward and reverse heat fluxes, respectively. ${ }^{32}$ Otey et al. proposed photon-mediated thermal rectification through vacuum, calculated near-field radiative heat transfer between SiC-3C and $\mathrm{SiC}-6 \mathrm{H}$ bulks, and predicted a maximum thermal rectification ratio of $0.41 .{ }^{3}$ Basu and Francoeur designed a near-field thermal rectifier using a doped silicon film and a doped silicon bulk and investigated the effects of the vacuum gap and the film thickness on rectification, realizing a rectification greater than 0.5 as a result. ${ }^{1}$ Yang et al. theoretically calculated that a thermal rectification ratio of almost 2 could be achieved utilizing bulk vanadium dioxide $\left(\mathrm{VO}_{2}\right)$ and silicon dioxide $\left(\mathrm{SiO}_{2}\right)$ separated by a $10 \mathrm{~nm}$ gap. The same work postulated that a rectification ratio of 3 can be attained with a $100 \mathrm{~nm}$ gap when bulk $\mathrm{SiO}_{2}$ is replaced by a thin film. ${ }^{33}$ The enhancing effects of the $\mathrm{VO}_{2}$ thin film and the $\mathrm{SiO}_{2}$ substrate on a near-field vacuum thermal switch have also been studied. ${ }^{34}$ Huang et al. designed a nearfield thermal rectification structure consisting of $\mathrm{VO}_{2}$ and $\mathrm{La}_{0.7} \mathrm{Ca}_{0.15} \mathrm{Sr}_{0.15} \mathrm{MnO}_{3}$ (LCSMO), which can achieve a rectification ratio of 7.7 with a vacuum gap of $10 \mathrm{~nm} .{ }^{35}$ Ghanekar et al. theoretically 
demonstrated that the thermal rectification ratio could be greatly enhanced to 16 in such cases by using one-dimensional (1D) rectangular and triangular $\mathrm{VO}_{2}$ surface gratings. ${ }^{4}$ Recently, Chen et al. studied an active-tuning near-field thermal rectifier using a stretchable structure composed of a polydimethylsiloxane (PDMS) thin film and a $\mathrm{VO}_{2}$ grating, which can achieve an ultrahigh rectification ratio of 23.7. ${ }^{5}$ Moreover, extensive research has achieved and further enhanced thermal rectification by utilizing various materials (both polar and phase-transition) and structures (plate-plate structures, micro/nanostructures, and surface gratings). ${ }^{9,36-47}$ Recently, Li et al. theoretically achieved rectification ratios over 140 with nanofilms of vanadium dioxide and cubic boron nitride ( $\mathrm{cBN}$ ) in the parallel plane geometry at the gap of $100 \mathrm{~nm}$ by leveraging the distinct scaling behaviors of the local density of states with film thicknesses for metals and insulators. ${ }^{48}$ Though many studies on the near-field radiative thermal rectification aim to further enhance the thermal rectification performance, the thermal rectification ratio has not been greatly improved.

In this Letter, we investigate the thermal rectification in a nearfield radiative thermal diode utilizing the phase-transition material $\mathrm{VO}_{2}$. To increase the thermal rectification ratio, different highinfrared-transmittance materials are employed, such as potassium bromide $(\mathrm{KBr})$, sodium chloride $(\mathrm{NaCl})$, polyethylene $(\mathrm{PE})$, and magnesium fluoride $\left(\mathrm{MgF}_{2}\right)$ as a thin-film substrate under the $\mathrm{VO}_{2}$ grating. Differing from previous studies that focus on the structure of the phase-transition material $\mathrm{VO}_{2}$, this study concentrates on the materials and structures of the substrate film. Our work indicates that the rectification ratio can be raised significantly by using a high-infraredtransmittance thin-film substrate (HITTS).

Here, we propose a near-field radiative thermal diode composed of a $\mathrm{VO}_{2} 1 \mathrm{D}$ grating and a HITTS. A typical design is shown in Fig. 1, incorporating two planar structures separated by a nanoscale distance less than the thermal wavelength $(1.8 \mu \mathrm{m} \leq \lambda \leq 14 \mu \mathrm{m})$. The passive

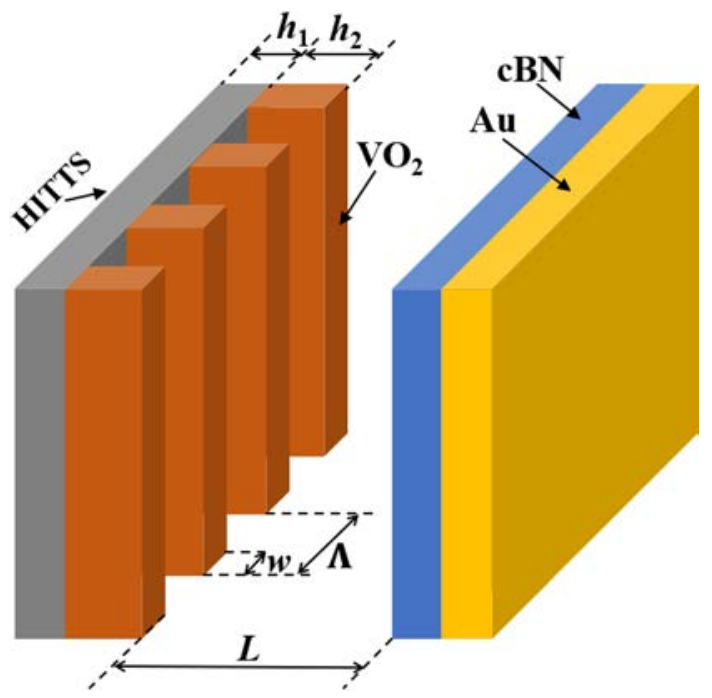

FIG. 1. Schematic of near-field thermal diodes using an infrared transparent film backsided phase-transition metasurface. The active side (left) top layer is a $1 \mathrm{D}$ rectangular grating made of $\mathrm{VO}_{2}$ with height $h_{2}$, width $w$, period $\Lambda$, and filling ratio $\phi=w / \Lambda$ and is deposited on a HITTS with thickness $h_{1}$. The passive counterpart (right) consists of a $1 \mu \mathrm{m}$ cBN layer atop a $1 \mu \mathrm{m}$ Au layer. side consists of a $1 \mu \mathrm{m}$ cubic boron nitride (cBN) layer on top of a $1 \mu \mathrm{m}$ gold $\mathrm{(Au}$ ) layer, which is kept constant throughout these analyses. The active counterpart is composed of a $\mathrm{VO}_{2}$ grating deposited on top of the HITTS. Four high-infrared-transmittance materials are considered within this work: $\mathrm{KBr}, \mathrm{NaCl}, \mathrm{PE}$, and $\mathrm{MgF}_{2}$.

To obtain radiative heat fluxes across the near-field thermal diode, we utilize the expression for NFRHT based on the dyadic Green's function formalism. ${ }^{49-52}$ The NFRHT expression is as follows:

$Q_{1 \rightarrow 2}\left(T_{1}, T_{2}, L\right)=\int_{0}^{\infty} \frac{d \omega}{2 \pi}\left[\Theta\left(\omega, T_{1}\right)-\Theta\left(\omega, T_{2}\right)\right] \int_{0}^{\infty} \frac{k_{\rho} d k_{\rho}}{2 \pi} \xi\left(\omega, k_{\rho}\right)$,

where $\Theta(\omega, T)=(\omega / 2) \operatorname{coth}\left(\omega / 2 k_{B} T\right)$ is the energy of the harmonic oscillator. The function $\int_{0}^{\infty} \frac{k_{\rho} d k_{\rho}}{2 \pi} \xi\left(\omega, k_{\rho}\right)$ is the spectral transmissivity of the radiative transport between media 1 and 2 separated by a distance $L$, where $\xi\left(\omega, k_{\rho}\right)$ is the energy transmission coefficient. Additionally, we use the effective medium approximation to obtain the effective dielectric properties of our proposed 1D grating structure. $^{53}$

To evaluate the effects of the HITTS on the thermal rectification performance of the diode described in Fig. 1, the heat flux is calculated based on the temperature difference between the active and passive sides of the diode $(2 \Delta T)$. Heat flux as a function of $2 \Delta T$ is plotted for the four high-infrared-transmittance material cases studied in Fig. 2. To evaluate the specific effects of the HITTS on performance, our study calculates the heat flux for a PDMS film-backed diode as a comparison. This provides a useful juxtaposition as PDMS has lower infrared transmittance than the other materials considered and has been recently studied in similar works. ${ }^{5}$ The temperature of the active side is $T_{1}=341 \mathrm{~K}+\Delta T$, while that of the passive side is set as $T_{2}=341 \mathrm{~K}-\Delta T$. This is chosen based on the metal-insulator-transition temperature of $\mathrm{VO}_{2}$,

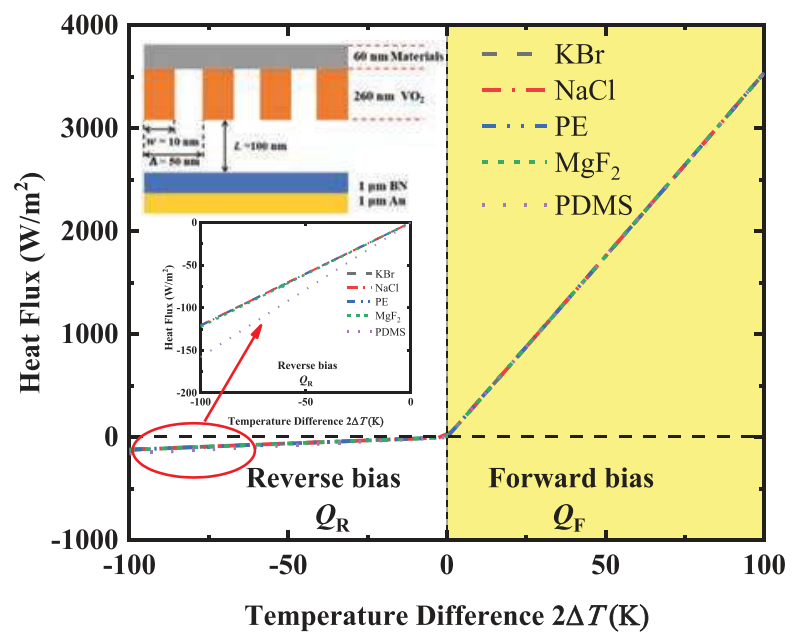

FIG. 2. Forward and reverse heat fluxes $\left(Q_{F}\right.$ and $\left.Q_{R}\right)$ as functions of the temperature difference between active and passive sides with a $100 \mathrm{~nm}$ gap for five different substrate materials, all with $h_{1}=60 \mathrm{~nm}, h_{2}=260 \mathrm{~nm}, \Lambda=50 \mathrm{~nm}, \phi=0.2$, and $L=100 \mathrm{~nm}$. 
which exists near $341 \mathrm{~K}$. As such, when $T_{1}>T_{2}$ (forward bias), $\mathrm{VO}_{2}$ is in the metallic phase; when $T_{1}<T_{2}$ (reverse bias), the $\mathrm{VO}_{2}$ layer is in the insulator phase. ${ }^{33}$ It can be clearly seen that the slopes of $Q_{F}$ are much larger than those of $Q_{R}$, indicating apparent diode-like characteristics. To illustrate why there is a strong asymmetry in the heat transport across the interfaces of the thermal diode, we plot the energy transmission coefficient $\xi\left(\omega, k_{\rho}\right)$ across the interfaces of the thermal diode with the $\mathrm{KBr}$ substrate at a gap of $100 \mathrm{~nm}$ shown in Fig. 3. Here, $k_{\rho} c / \omega$ is the normalized parallel wavevector. Transmission is high at two prominent frequencies that occur near the characteristic wavelengths of cBN (7.6 and $9.8 \mu \mathrm{m})$. Since metallic $\mathrm{VO}_{2}$ does not support surface phonon polariton in the infrared wavelength region, the NFRHT is mainly due to the symmetric and antisymmetric surface phonon polaritons supported by the $\mathrm{cBN}$ layer. Aside from that, there is a weak contribution based on the Fabry-Pérot modes and frustrated modes $\left(k_{\rho} c / \omega \approx 1\right)$ in the near-field regime. High energy transmission is attributed to the tunneling of surface waves across interfaces when the diode is in the forward bias. However, the NFRHT is dominated by non-resonant surface waves when the diode is in the reverse bias because $\mathrm{cBN}$ and insulator $\mathrm{VO}_{2}$ support the nonoverlapping surface phonon modes. Meanwhile, the surface phonons of insulator $\mathrm{VO}_{2}$ appear in the frequency range, where the extinction coefficient of $\mathrm{cBN}$ is very low $(\kappa \approx 0)$. Therefore, tunneling between $\mathrm{cBN}$ and insulator $\mathrm{VO}_{2}$ is much weaker than that of $\mathrm{cBN}$ and metallic $\mathrm{VO}_{2}$, which contributes to the strong asymmetry in the heat transport across the interfaces of the thermal diode. The diodes backed with high-infrared-transmittance materials $\left(\mathrm{KBr}, \mathrm{NaCl}, \mathrm{PE}\right.$, or $\left.\mathrm{MgF}_{2}\right)$ on the active side realize improved thermal rectification performance with respect to the PDMS-backed diode. This is because HITTS cannot absorb more infrared spectrum radiation emitted from the passive side when the diode is in the reverse bias shown in the inset of Fig. 2. These data prove that the transmission properties of the thin film substrate play an important role in the radiative heat transfer across the diode.

(a)

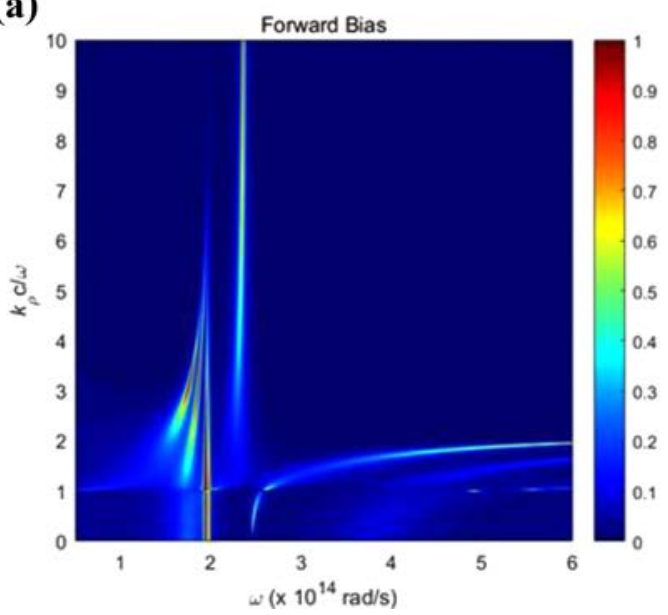

In order to further illustrate the effects of different highinfrared-transmittance materials as the substrate on the thermal rectification performance of the diode, we analyze the impact all the related geometric parameters on the rectification ratio of the near-field radiative thermal diode, including the thickness of the substrate film $h_{1}$, the height of the $\mathrm{VO}_{2}$ grating $h_{2}$, the filling ratio $\phi$, and the gap $L$. The rectification ratios are calculated when the temperature of the active side is set as $351 \mathrm{~K}$ for the forward bias and $331 \mathrm{~K}$ for the reverse bias, and that of the passive side is kept constant at $341 \mathrm{~K}$. Figure 4 (a) shows the rectification ratio $R$ vs the filling ratio of the $\mathrm{VO}_{2}$ grating for different HITTSs. It is evident that when the value of the filling ratio $\phi$ decreases from 1.0 to 0.1 , these rectification ratios trend upwards with varying slopes. A sharp decrease in the rectification ratio is noted when the value of filling ratio $\phi$ increases from 0.1 to 0.3 . This trend proves that the filling ratio greatly affects the rectification ratio of the diode, because the filling ratio directly affects the optical characteristics of the $\mathrm{VO}_{2}$ grating structure, resulting in changes in the surface waves across the interfaces. It is also easy to understand that the $\mathrm{VO}_{2}$ grating behaves as a thin film with a relatively weaker thermal rectification when the filling ratio approaches to 1 , which has been proved in Ref. 4 . The rectification ratio will continue to grow when the filling ratio further decreases $(\phi<0.1)$, but it is impractical as the grating period is $\Lambda=50 \mathrm{~nm}$. Meanwhile, it is apparent that the rectification ratio curves for HITTS cases are above those of PDMS cases for all values of the filling ratio, deviating most notably at low values of $\phi$. Therefore, it can be concluded that the HITTS can enhance the thermal rectification performance with respect to other substrates.

To illustrate how other geometric parameters affect the thermal rectification of the diode, Fig. 4 (b) provides results for the rectification ratio $R$ simulated with different $\mathrm{VO}_{2}$ grating heights $\left(h_{2}\right)$. For the four HITTSs, the rectification ratio sharply decreases as the grating height $h_{2}$ increases from 10 to $100 \mathrm{~nm}$. Conversely, the PDMS film rectification ratio trends upwards with gradually decreasing slopes. Here, we plot spectral heat flux $d q / d \lambda$ in forward and reverse biases for $\mathrm{KBr}$ and

(b)

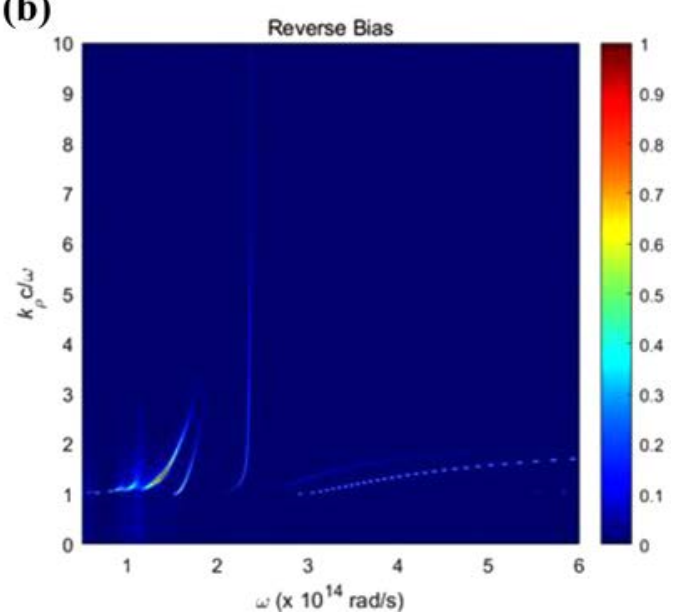

FIG. 3. Coefficient of energy transmission $\xi\left(\omega, k_{\rho}\right)$ across the two interfaces of an ultrahigh-rectification thermal diode with the KBr substrate against angular frequency $\omega$ and normalized parallel wavevector $k_{\rho} c / \omega$ for the (a) forward bias and (b) reverse bias. 

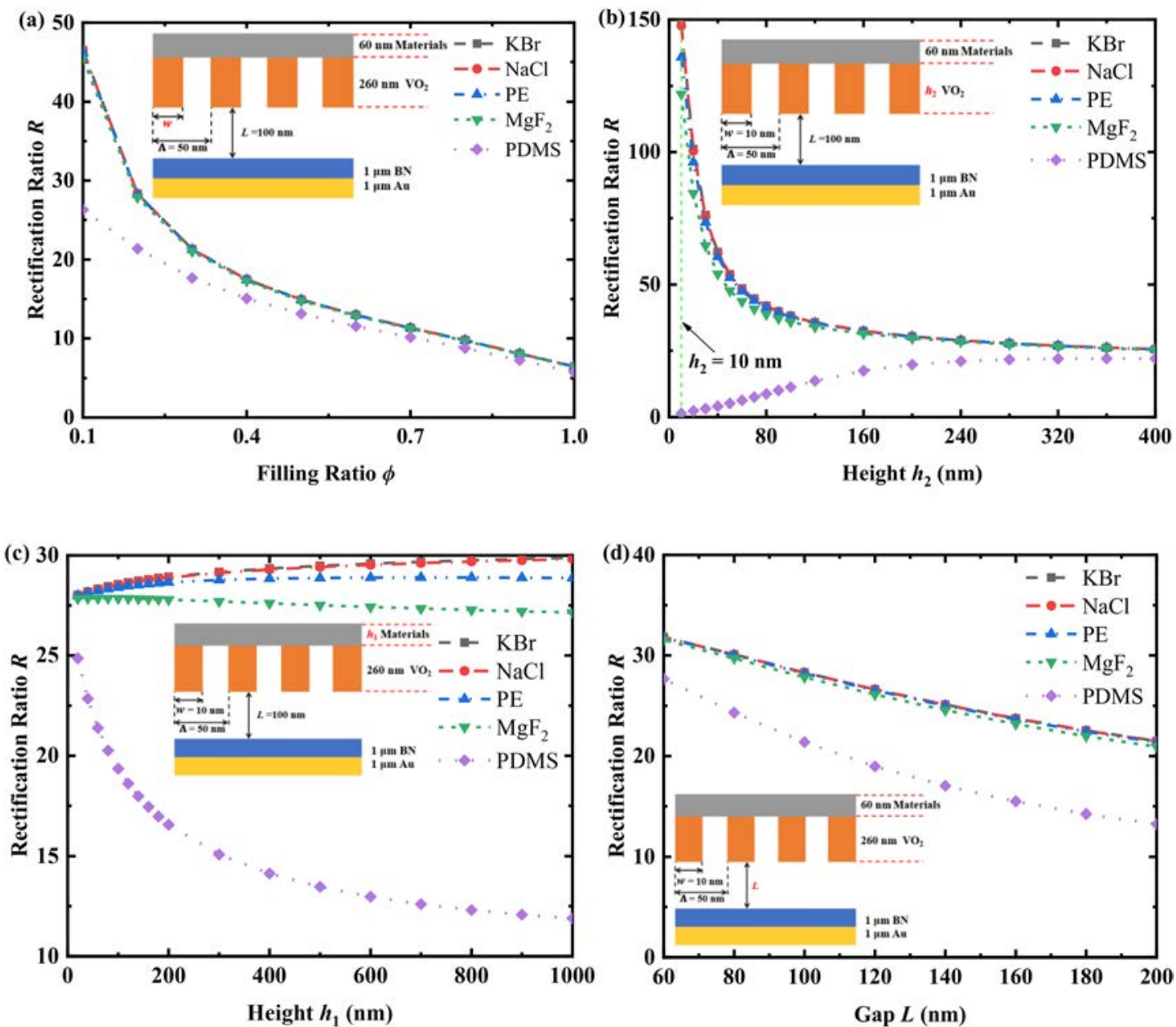

FIG. 4. Rectification ratio $R$ as a function of the following geometric parameters: (a) filling ratio $\phi$, (b) height of $\mathrm{VO}_{2}$ grating $h_{2}$, (c) thickness of the substrate film $h_{1}$, and (d) gap $L$. For all parameters not varied in each figure, the following constant values are used: $h_{1}=60 \mathrm{~nm}, h_{2}=260 \mathrm{~nm}, \Lambda=50 \mathrm{~nm}, \phi=0.2$, and $L=100 \mathrm{~nm}$. The temperature of the active side is set as $351 \mathrm{~K}$ for the forward bias and $331 \mathrm{~K}$ for the reverse bias, and that of the passive side is kept constant at $341 \mathrm{~K}$.

PDMS cases with different $\mathrm{VO}_{2}$ grating heights, including 20, 40, 60, 80, and $100 \mathrm{~nm}$, at a gap of $100 \mathrm{~nm}$ shown in Fig. 5. The reason for a pair of opposite trends is that the increase in the grating height can improve the radiation absorption emitted from the passive side when the diode is in the reverse bias for HITTS cases shown in Fig. 5(a), while the optical properties of the PDMS film can cause the two prominent absorption peaks around the 7.6 and $9.8 \mu \mathrm{m}$ to descend with the increase in the height of $\mathrm{VO}_{2}$ grating shown in Fig. 5(b), resulting in the decrease in the overall heat flux in the reverse bias. When $h_{2}$ reaches a certain threshold (approximately greater than $200 \mathrm{~nm}$ ), the rectification ratios become quite similar for all different substrate materials. This indicates that the HITTS affects the thermal rectification of diodes to a much greater degree when the $\mathrm{VO}_{2}$ grating height is significantly smaller than $100 \mathrm{~nm}$.

Next, Fig. 4(c) shows the thermal rectification ratios calculated vs the thickness of the thin film substrate $h_{1}$ for the five different materials mentioned above. For HITTSs made by $\mathrm{KBr}$ and $\mathrm{NaCl}$, respectively, an increase in film thickness $h_{1}$ leads to a slow increase in the rectification ratio. In the case of the $\mathrm{PE}$ or $\mathrm{MgF}_{2}$ backing, the slope remains nearly the same for all values of the film thickness. On the other hand, the rectification ratio for the PDMS-backed diode trends downwards with different slopes as $h_{1}$ increases. Because the increased thickness of the PDMS thin film substrate can enhance the radiation absorption of the active side when the diode is in the reverse bias, while HITTSs cannot achieve it as mentioned above. Thus, we can conclude that compared with the PDMS film backing, the change in the thickness of HITTS $h_{1}$ has little impact on the thermal rectification of the diode.

Figure 4(d) displays the rectification ratio as a function of the diode gap $L$ for five cases with different substrate films. It can be seen clearly that $R$ is inversely proportional to the gap $L$. This is because surface waves gradually become more dominant at smaller gaps, and vice versa.

Based on the above analysis of design parameters, the highest performing values for the four parameters are determined: $h_{1}=1 \mu \mathrm{m}$, $\Lambda=50 \mathrm{~nm}, \phi=0.2$, and $L=100 \mathrm{~nm}$. With these values, and by 

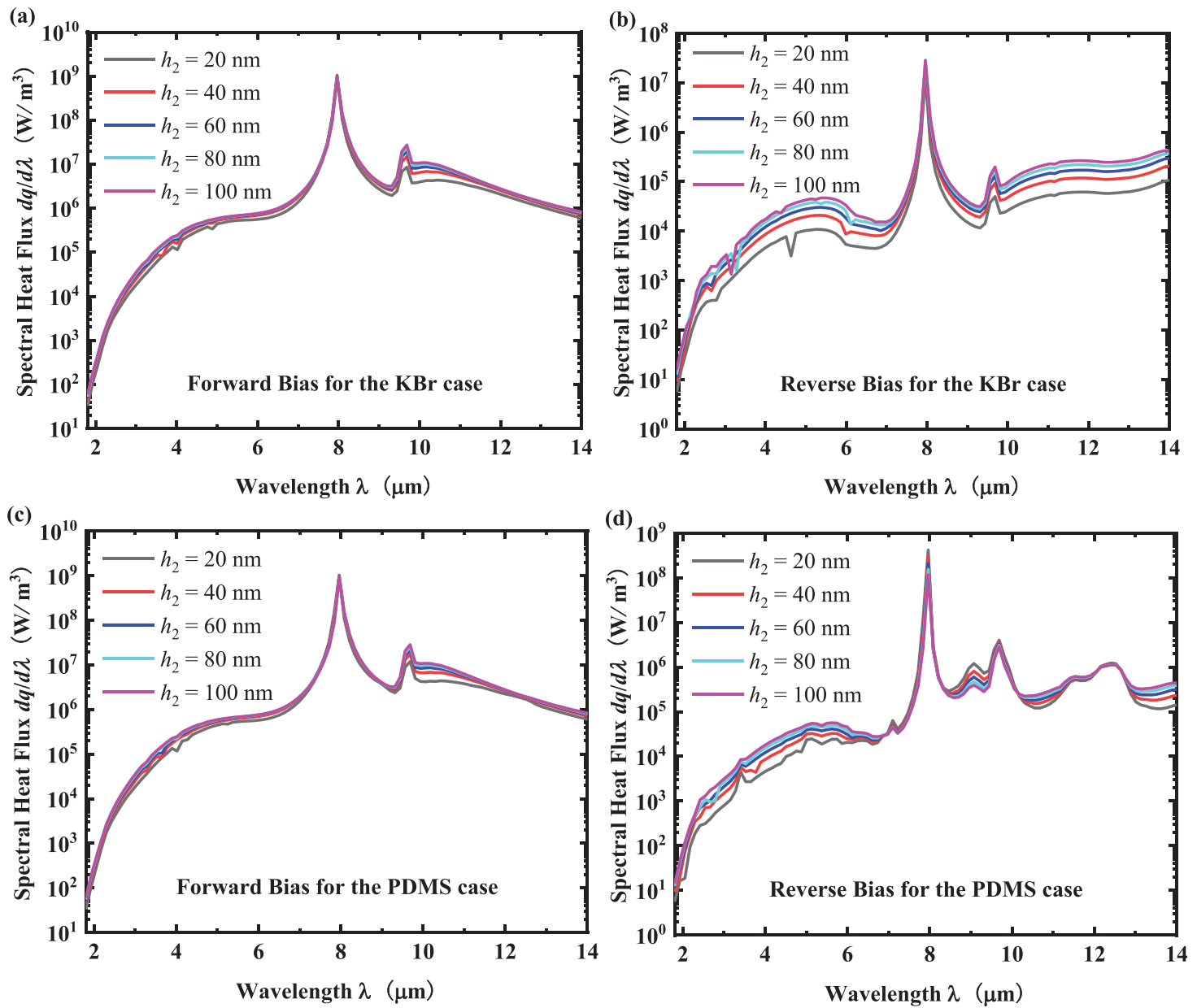

FIG. 5. Comparison of spectral heat fluxes in forward and reverse biases for $\mathrm{KBr}$ and $\mathrm{PDMS}$ thin-film substrates with different $\mathrm{VO}_{2}$ grating heights, including 20 , $40,60,80$, and $100 \mathrm{~nm}$. (a) KBr case: forward bias, (b) KBr case: reverse bias, (c) PDMS case: forward bias, and (d) PDMS case: reverse bias.

optimizing the height of the $\mathrm{VO}_{2}$ grating $h_{2}$, a greatly increased diode rectification ratio can be achieved, as shown in Fig. 6. It is apparent that, for the HITTS, the rectification ratio increases with a decrease in the height of the $\mathrm{VO}_{2}$ grating. Conversely, for the PDMS case, the rectification ratio decreases with the decrease in $h_{2}$. The rectification ratios for the HITTS cases are also much larger than those of the PDMS case. The rectification ratio reaches a maximum value of $R=161$ when the $\mathrm{VO}_{2}$ grating height is reduced to $h_{2}=10 \mathrm{~nm}$ for the $\mathrm{KBr}$-backed diode. While the value of rectification ratio $R$ can reach a higher value when $h_{2}<10 \mathrm{~nm}$, the $\mathrm{VO}_{2}$ grating height $h_{2}<10 \mathrm{~nm}$ is impractical for the nanofabrication process. Therefore, considering the above comprehensive analysis of design parameters and the feasibility of nanofabrication, a set of optimal parameters is determined: $h_{1}=1 \mu \mathrm{m}, h_{2}=10 \mathrm{~nm}, \Lambda=50 \mathrm{~nm}, \phi=0.2$, and $L=100 \mathrm{~nm}$. By utilizing a $1 \mathrm{D}$ grating made of the phase-transition material $\mathrm{VO}_{2}$ and a thin-film substrate made of high-infrared-transmittance material $\mathrm{KBr}$, an ultrahigh rectification ratio of 161 can be obtained-a substantial increase compared with previous studies.
In summary, this theoretical investigation proves that highly efficient thermal rectification can be achieved using the $1 \mathrm{D} \mathrm{VO}_{2}$ grating metasurface backed with a large variety of high-infrared-transmittance thin-film substrates. This study shows that the design parameters of the thermal diode play an incredibly important role in enhancing rectification, such as the filling ratio, the height of the $\mathrm{VO}_{2}$ grating, the thickness of the substrate film, and the selection of substrate materials. Through the study of four materials $(\mathrm{KBr}, \mathrm{NaCl}, \mathrm{PE}$, and $\mathrm{MgF}_{2}$ ), we conclude that thin film substrates composed of highinfrared-transmittance materials such as these can greatly promote the thermal rectification rate of a diode. With optimized geometric parameters and a thin film substrate made of $\mathrm{KBr}$, an ultrahigh rectification ratio of $161 \mathrm{can}$ be obtained at a gap of $100 \mathrm{~nm}$. This work verifies that employing a highly infrared transmissive thin film as the active side substrate plays a significant role in the enhancement of the near-field thermal rectification of a diode. The data and analyses shed light on high performance thermal rectification devices, which can be leveraged for small-scale thermal management in the future. 


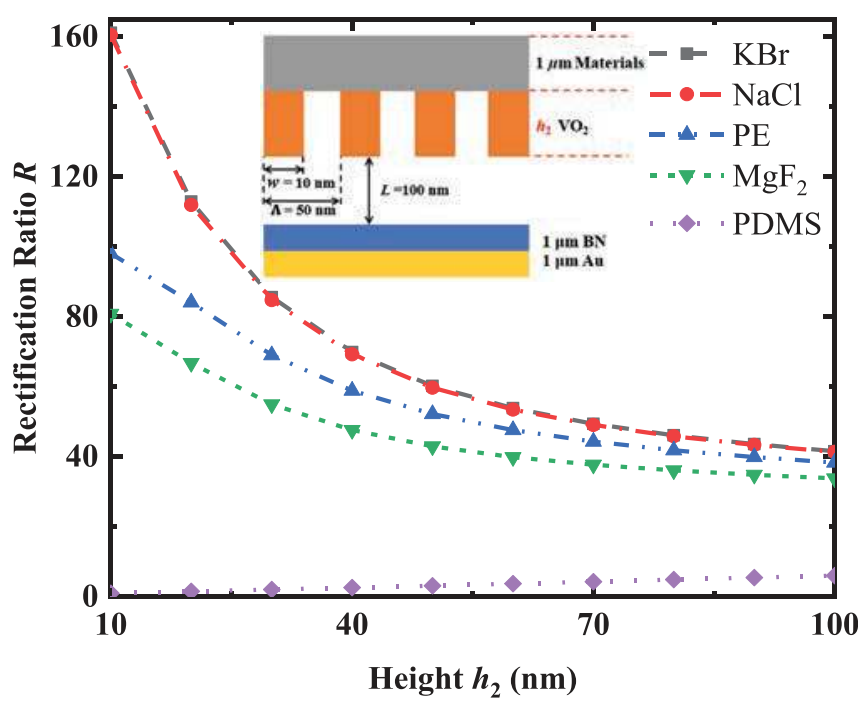

FIG. 6. Effect of $\mathrm{VO}_{2}$ grating height $h_{2}$ on the rectification ratio $R$ when $h_{1}=1 \mu \mathrm{m}$, $\Lambda=50 \mathrm{~nm}, \phi=0.2$, and $L=100 \mathrm{~nm}$.

This work was supported by the National Science Foundation (No. CBET-1941743).

\section{DATA AVAILABILITY}

The data that support the findings of this study are available from the corresponding author upon reasonable request.

\section{REFERENCES}

${ }^{1}$ S. Basu and M. Francoeur, Appl. Phys. Lett. 98, 113106 (2011).

${ }^{2}$ T. Ruokola, T. Ojanen, and A. P. Jauho, Phys. Rev. B 79, 144306 (2009).

${ }^{3}$ C. R. Otey, W. T. Lau, and S. Fan, Phys. Rev. Lett. 104, 154301 (2010).

${ }^{4}$ A. Ghanekar, J. Ji, and Y. Zheng, Appl. Phys. Lett. 109, 123106 (2016).

${ }^{5}$ F. Q. Chen, X. J. Liu, Y. P. Tian, and Y. Zheng, Adv. Eng. Mater. 23, 2000825 (2020).

${ }^{6}$ P. Ben-Abdallah and S. A. Biehs, Appl. Phys. Lett. 103, 191907 (2013).

${ }^{7}$ Z. Chen, C. Wong, S. Lubner, S. Yee, J. Miller, W. Jang, C. Hardin, A. Fong, J.

E. Garay, and C. Dames, Nat. Commun. 5, 5446 (2014).

${ }^{8}$ P. Ben-Abdallah and S. A. Biehs, AIP Adv. 5, 053502 (2015).

${ }^{9}$ P. Ben-Abdallah and S. A. Biehs, Phys. Rev. Lett. 112, 044301 (2014).

${ }^{10}$ W. Kobayashi, Y. Teraoka, and I. Terasaki, Appl. Phys. Lett. 95, 171905 (2009).

${ }^{11}$ T. Takeuchi, H. Goto, R. S. Nakayama, Y. I. Terazawa, K. Ogawa, A. Yamamoto, T. Itoh, and M. Mikami, J. Appl. Phys. 111, 093517 (2012).

${ }^{12}$ D. Sawaki, W. Kobayashi, Y. Moritomo, and I. Terasaki, Appl. Phys. Lett. 98, 081915 (2011).

${ }^{13}$ W. Kobayashi, D. Sawaki, T. Omura, T. Katsufuji, Y. Moritomo, and I. Terasaki, Appl. Phys. Express 5, 027302 (2012).

${ }^{14}$ M. Martínez-Pérez, A. Fornieri, and F. Giazotto, Nat. Nanotechnol. 10, 303 (2015).

${ }^{15}$ D. Polder and M. V. Hove, Phys. Rev. B 4, 3303 (1971).

${ }^{16}$ A. I. Volokitin and B. N. J. Persson, Rev. Mod. Phys. 79, 1291 (2007).

${ }^{17}$ J. L. Song, Q. Cheng, B. Zhang, L. Lu, and R. Hu, Rep. Prog. Phys. 84, 036501 (2021).

${ }^{18}$ Y. M. Xuan, Photonics Nanostruct.-Fundam. Appl. 12, 93 (2014).
${ }^{19}$ B. Song, D. Thompson, A. Fiorino, Y. Ganjeh, P. Reddy, and E. Meyhofer, Nat. Nanotechnol. 11, 509 (2016).

${ }^{20}$ A. Fiorino, D. Thompson, L. Zhu, B. Song, P. Reddy, and E. Meyhofer, Nano Lett. 18, 3711 (2018)

${ }^{21}$ M. Lim, J. Song, S. S. Lee, and B. J. Lee, Nat. Commun. 9, 4302 (2018).

${ }^{22}$ J. DeSutter, L. Tang, and M. Francoeur, Nat. Nanotechnol. 14, 751 (2019).

${ }^{23}$ S. Basu, Z. M. Zhang, and C. J. Fu, Int. J. Energy Res. 33, 1203 (2009).

${ }^{24}$ A. Narayanaswamy and G. Chen, Phys. Rev. B 77, 075125 (2008).

${ }^{25}$ S. Shen, A. Narayanaswamy, and G. Chen, Nano Lett. 9, 2909 (2009).

${ }^{26}$ L. Tang, J. Desutter, and M. Francoeur, ACS Photonics 7, 1304 (2020)

${ }^{27}$ B. Song, Y. Ganjeh, S. Sadat, D. Thompson, A. Fiorino, V. Fernández-Hurtado, J. Feist, F. J. Garcia-Vidal, J. C. Cuevas, and P. Reddy, Nat. Nanotechnol. 10, 253 (2015).

${ }^{28}$ K. Kim, B. Song, V. Fernández-Hurtado, W. Lee, W. Jeong, L. Cui, D. Thompson, J. Feist, M. T. H. Reid, F. J. García-Vidal, J. C. Cuevas, E. Meyhofer, and P. Reddy, Nature 528, 387 (2015).

${ }^{29}$ F. Yang and B. Song, Phys. Rev. B 103, 235415 (2021).

${ }^{30}$ W. Gu, G. H. Tang, and W. Q. Tao, Int. J. Heat Mass Transfer 82, 429 (2015).

${ }^{31}$ V. Kubytskyi, S. A. Biehs, and P. Ben-Abdallah, Phys. Rev. Lett. 113, 074301 (2014).

${ }^{32}$ B. Song, A. Fiorino, E. Meyhofer, and P. Reddy, AIP Adv. 5, 053503 (2015).

${ }^{33}$ Y. Yang, S. Basu, and L. Wang, Appl. Phys. Lett. 103, 163101 (2013).

${ }^{34}$ Y. Yang, S. Basu, and L. Wang, J. Quant. Spectrosc. Radiat. Transfer 158, 69 (2015).

${ }^{35}$ J. Huang, L. Qiang, Z. Zheng, and Y. Xuan, Int. J. Heat Mass Transfer 67, 575 (2013).

${ }^{36}$ R. Audhkhasi and M. L. Povinelli, J. Appl. Phys. 126, 063106 (2019).

${ }^{37}$ G. Xu, J. Sun, H. Mao, and T. Pan, J. Appl. Phys. 124, 183104 (2018).

${ }^{38}$ A. Didari, E. B. Eliolu, T. Okutucu-Özyurt, and M. P. Mengüç, J. Quant. Spectrosc. Radiat. Transfer 212, 120 (2018).

${ }^{39}$ J. Shen, X. Liu, H. He, W. Wu, and B. Liu, J. Quant. Spectrosc. Radiat. Transfer 211, 1-8 (2018).

${ }^{40}$ L. P. Wang and Z. M. Zhang, Nanoscale Microscale Thermophys. Eng. 17, 337 (2013).

${ }^{41}$ K. Ito, K. Nishikawa, H. Iizuka, and H. Toshiyoshi, Appl. Phys. Lett. 105, 253503 (2014).

${ }^{42}$ A. M. Morsy, M. T. Barako, V. Jankovic, V. D. Wheeler, M. W. Knight, G. T. Papadakis, L. A. Sweatlock, P. Hon, and M. L. Povinelli, Sci. Rep. 10, 13964 (2020).

${ }^{43}$ F. Q. Chen, X. J. Liu, Y. P. Tian, D. Y. Wang, and Y. Zheng, J. Quant. Spectrosc. Radiat. Transfer 259, 107414 (2021).

${ }^{44}$ A. Ghanekar, Y. Tian, M. Ricci, S. Zhang, and Z. Yi, Opt. Express 26, A209 (2018).

${ }^{45}$ Y. Zhang, C. L. Zhou, H. L. Yi, and H. P. Tan, Phys. Rev. Appl. 13, 034021 (2020).

${ }^{46}$ C. L. Zhou, L. Qu, Y. Zhang, and H. L. Yi, J. Quant. Spectrosc. Radiat. Transfer 251, 107023 (2020)

${ }^{47}$ P. Zhang, P. Yang, Z. Zheng, and W. Yu, Int. J. Heat Mass Transfer 155, 119707 (2020).

${ }^{48}$ Q. Z. Li, H. Y. He, Q. Chen, and B. Song, Phys. Rev. Appl. 16, 014069 (2021).

${ }^{49}$ X. L. Liu and Z. M. Zhang, Appl. Phys. Lett. 104, 251911 (2014).

${ }^{50}$ X. L. Liu, B. Zhao, and Z. M. Zhang, Phys. Rev. A 91, 062510 (2015).

${ }^{51}$ A. Narayanaswamy and Y. Zheng, J. Quant. Spectrosc. Radiat. Transfer 132, 12 (2014).

${ }^{52}$ J. Lussange, R. Guérout, F. S. S. Rosa, J. J. Greffet, A. Lambrecht, and S. Reynaud, Phys. Rev. B 86, 085432 (2012).

${ }^{53}$ E. N. Glytsis and T. K. Gaylord, Appl. Opt. 31, 4459 (1992).

${ }^{54}$ D. H. Raguin and G. M. Morris, Appl. Opt. 32, 1154 (1993).

${ }^{55} \mathrm{P}$. Lalanne and D. Lemercier-Lalanne, J. Mod. Opt. 43, 2063 (1996).

${ }^{56}$ Y. B. Chen, Z. M. Zhang, and P. J. Timans, J. Heat Transfer 129, 79 (2007).

${ }^{57}$ X. L. Liu, T. J. Bright, and Z. M. Zhang, J. Heat Transfer 136, 092703 (2014). 